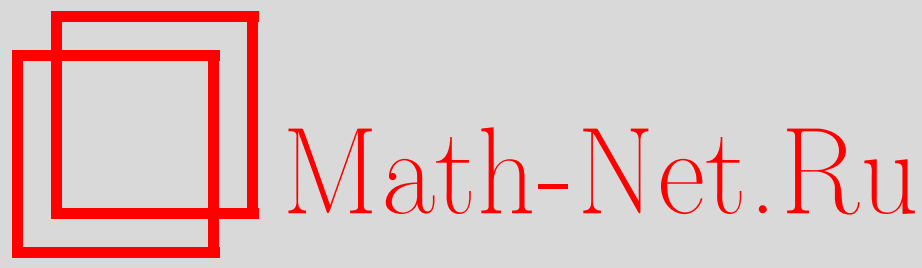

В. Д. Ляховский, Свойства максимумов функции кратности, ТМФ, 2013, том 174, номер 1, 99-108

DOI: https://doi.org/10.4213/tmf8354

Использование Общероссийского математического портала Math-Net.Ru подразумевает, что вы прочитали и согласны с пользовательским соглашением http: //www.mathnet.ru/rus/agreement

Параметры загрузки :

IP: 54.162 .27 .143

26 апреля 2023 г., 11:50:41

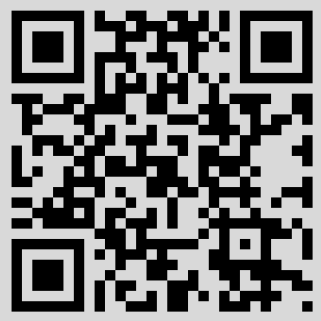




\title{
СВОЙСТВА МАКСИМУМОВ ФУНКЦИИ КРАТНОСТИ
}

\begin{abstract}
Функция кратности $M(\nu, p)$ в разложении тензорной степени алгебры $\left(L^{\omega}\right)^{\otimes p}$ переопределена как гладкая функция в весовом пространстве $P_{\mathfrak{g}}$ алгебры Ли g. Изучается поведение максимумов функции кратности. В результате для любой фиксированной степени $p$ можно легко найти подмодуль с максимальной кратностью.
\end{abstract}

Ключевые слова: алгебры Ли, теория представлений, тензорные степени, интегрируемые модели.

DOI: $10.4213 / \operatorname{tmf} 8354$

\section{1. ВВЕДЕНИЕ}

В квантовых моделях (таких, как интегрируемые системы, конформные теории поля и квантовая гравитация) чрезвычайно важной является проблема разложения тензорного произведения. Спиновые цепочки представляют собой естественные примеры таких систем. Если имеется $\mathfrak{g}$-инвариантность, то компоненты преобразуются как $\mathfrak{g}$-модули $L^{\omega}$ со старшими весами, при этом состояния системы ведут себя как $\left(L^{\omega}\right)^{\otimes p}$ со старшим весом $p \omega$. Структура спектра гамильтониана определяется кратностями $m(\nu, p)$ в разложении

$$
\left(L^{\omega}\right)^{\otimes p}=\sum_{\nu} m(\nu, p) L^{\nu}
$$

В таком контексте $\mathfrak{g}$ является простой классической алгеброй Ли, а $L^{\omega}-$ фундаментальным g-модулем минимальной размерности (со старшим весом $\omega$ ). Мы можем рассматривать $\left(L^{\omega}\right)^{\otimes p}$ как $\oplus^{p} \mathfrak{g}$-неприводимый модуль, а разложение считать редукцией алгебры $\oplus^{p} \mathfrak{g}$ к диагональной подалгебре в ней.

Основной задачей так называемой проблемы тензорных степеней является определение всех компонент разложения вместе с их кратностями [1]. Существует множество комбинаторных исследований этой проблемы (см., например, статьи [2] и [3]), а также ряд работ, связанных с фермионными формулами (например, статьи [4]-[6]). На этом пути были разработаны различные комбинаторные методы и получены важные результаты общего характера. Практические вычисления с использованием указанных комбинаторных формул едва ли возможны во всех случаях, за исключением простейших примеров [7].

${ }^{*}$ Санкт-Петербургский государственный университет, Санкт-Петербург, Россия. E-mail: lyakh1507@nm.ru 
В настоящей статье мы рассматриваем кратности $M(\nu, p)$ в разложении $(1)$ как функции, параметризованные параметром $p$ и определенные на всем весовом пространстве: $\nu \in P_{\mathfrak{g}}$. Будучи ограниченной на замкнутую фундаментальную камеру Вейля $\overline{C^{(0)}}$, функция кратности совпадает с соответствующими коэффициентами кратности:

$$
M^{\otimes^{p} \omega}(\nu, p) \downarrow \frac{}{C^{(0)}}=m^{\otimes^{p} \omega}(\nu, p) .
$$

Используя свойства симметрии Вейля, мы найдем явные общие решения для последовательностей $A$ и $B$ [8], [9].

\section{2. ОБЩИЕ СВОЙСТВА ФУНКЦИЙ КРАТНОСТИ}

Кратко напомним рекуррентные свойства функций кратности. Рассмотрим формулу Вейля в терминах формальных характеров $\operatorname{ch}(L)$ для тензорного произведения $L^{\mu} \otimes L^{\nu}$, интерпретируемого как модуль диагональной подалгебры $\mathfrak{g}_{\text {diag }} \rightarrow \mathfrak{g} \oplus \mathfrak{g}$,

$$
\operatorname{ch}\left(L^{\mu}\right) \operatorname{ch}\left(L^{\nu}\right)_{\downarrow P_{\text {diag }}^{+}}=\sum_{\xi \in P_{\text {diag }}} M_{\xi}^{\mu \nu} \operatorname{ch}\left(L^{\xi}\right) .
$$

Ее можно переписать через сингулярные элементы $\Psi^{(\nu)}=\sum_{w \in W} \epsilon(w) e^{(\nu+\rho)}$ (здесь $\epsilon(w)$ - детерминант оператора $w$, действующего на $\left.P_{\mathfrak{g}}\right)$ :

$$
\left(\frac{\Psi^{(\mu)} \Psi^{(\nu)}}{\Psi^{(0)} \Psi^{(0)}}\right)_{\downarrow P_{\text {diag }}}=\sum_{\xi \in P_{\text {diag }}} M_{\xi}^{\mu \nu} \frac{\Psi_{\text {diag }}^{(\xi)}}{\Psi_{\text {diag }}^{(0)}} .
$$

Произведение в формальной алгебре коммутирует с операцией проектирования:

$$
\left(\Psi^{(\mu)} \Psi^{(\nu)}\right)_{\downarrow P_{\text {diag }}}=\Psi_{\downarrow P_{\text {diag }}}^{(\mu)} \Psi_{\downarrow P_{\text {diag }}}^{(\nu)}=\Psi_{\text {diag }}^{(\mu)} \Psi_{\text {diag }}^{(\nu)} .
$$

Таким образом, один множитель можно исключить, а другой преобразует соответствующий сингулярный элемент в весовую диаграмму модуля, скажем, $N\left(L_{\mathrm{diag}}^{(\nu)}\right)$ :

$$
\begin{aligned}
\left(\Psi_{\text {diag }}^{(0)}\right)^{-1} \Psi_{\text {diag }}^{(\mu)} \Psi_{\text {diag }}^{(\nu)} & =\sum_{\xi \in P_{\text {diag }}} M_{\xi}^{\mu \nu} \Psi_{\text {diag }}^{(\xi)}= \\
& =N\left(L_{\text {diag }}^{(\mu)}\right) \Psi_{\text {diag }}^{(\nu)}=\Psi_{\text {diag }}^{(\mu)} N\left(L_{\text {diag }}^{(\nu)}\right) .
\end{aligned}
$$

В результате на функции кратности $M(\nu, p)$ налагается система связей [10], [11]:

$$
M_{\xi}^{\otimes^{p} \omega}-M_{\xi-\omega}^{\otimes^{(p-1)} \omega}=M_{\xi-\omega+\alpha_{1}}^{\otimes^{(p-1)} \omega}+M_{\xi-\omega+\alpha_{1}+\alpha_{2}}^{\otimes^{(p-1)} \omega}+\cdots+M_{\xi-\omega+\alpha_{1}+\alpha_{2}+\cdots+\alpha_{n}}^{\otimes^{(p-1)} \omega}
$$

Для решения рекуррентных соотношений используются свойства симметрии Вейля. В работах [8], [9] доказано, что общие решения для последовательностей $A$ и $B$ имеют вид

$$
\begin{aligned}
& M_{A_{n}}^{\otimes^{p} \omega}\left(\nu\left\{a_{i}\right\}\right)=\frac{p ! \prod_{i=1}^{n} a_{i} \prod_{j=1}^{n-1}\left(a_{j}+a_{j+1}\right) \ldots \prod_{j=1}^{2}\left(\sum_{k=1}^{n-1} a_{j+k}\right)\left(\sum_{k=1}^{n} a_{k}\right)}{\prod_{l=0}^{n}\left(\frac{1}{n+1}\left(p+\frac{n(n+1)}{2}+\sum_{s=1}^{l}\left(-s a_{s}\right)+\sum_{t=l}^{n-1}(n-t) a_{t+1}\right)\right) !} \\
& M_{B_{n}}^{\otimes^{p} \omega}\left(\nu\left\{a_{i}\right\}\right)=\prod_{k=0}^{n-1} \frac{(p+2 k) ! \prod_{l=1}^{n} a_{l} \prod_{i<j}\left(a_{i}^{2}-a_{j}^{2}\right)}{2^{2 k}\left(\frac{p+a_{k+1}+2 n-1}{2}\right) !\left(\frac{p-a_{k+1}+2 n-1}{2}\right) !} .
\end{aligned}
$$


Здесь $M^{\otimes^{p} \omega}$ - функции координат $a_{i}$ старшего веса $\nu$ в базисе фундаментальных весов, измеренных от центральной точки симметрии Вейля: $\nu+\rho=\sum_{i} a_{i} \omega_{i}$ (в случае $B_{n}$ рассматриваются координаты в базисе $\left.\left\{e_{i} / 2\right\}\right)$. В частности, для алгебр $A_{2}$ и $B_{2}$ и старшего веса $\nu+\rho=a_{1} \omega_{1}+a_{2} \omega_{2}$ получим следующие компактные выражения:

$$
\begin{aligned}
& M_{A_{2}}\left(p ;\left\{a_{1}, a_{2}\right\}\right)=\frac{p ! a_{1} a_{2}\left(a_{1}+a_{2}\right)}{\left(\frac{p-\left(a_{1}+2 a_{2}\right)+3}{3}\right) !\left(\frac{p+\left(2 a_{1}+a_{2}\right)+3}{3}\right) !\left(\frac{p+\left(-a_{1}+a_{2}\right)+3}{3}\right) !}, \\
& M_{B_{2}}\left(p ;\left\{a_{1}, a_{2}\right\}\right)=\frac{p !(p+2) ! a_{1} a_{2}\left(a_{1}+a_{2}\right)\left(a_{1}-a_{2}\right)}{4\left(\frac{p+a_{1}+3}{2}\right) !\left(\frac{p+a_{2}+3}{2}\right) !\left(\frac{p-a_{1}+3}{2}\right) !\left(\frac{p-a_{2}+3}{2}\right) !} .
\end{aligned}
$$

Явные выражения для функций кратности дают нам уникальную возможность изучения свойств сложных квантовых систем. Например, в специальной системе координат зависимость функции кратности от $p$ становится полиномиальной. Пусть центр новой системы для алгебры $\mathfrak{g}=A_{2}$ находится на конце старшего веса $(p+$ 1) $\omega_{1}+\omega_{2}$. Положим

$$
a_{1}=\frac{1}{2}\left(2 p-3 b_{1}-b_{2}+2\right), \quad a_{2}=b_{2}+1 .
$$

В координатах $\left\{b_{j}\right\}$ функция кратности имеет вид

$$
\begin{aligned}
M_{A_{2}}\left(p ; b_{1}, b_{2}\right)= & \frac{p !}{\left(p-b_{1}+2\right) !}\left(2 p-3 b_{1}-b_{2}+2\right)\left(2 p-3 b_{1}+b_{2}+4\right) \times \\
& \times \frac{1}{4} \frac{b_{2}+1}{\left(\frac{b_{1}-b_{2}}{2}\right) !\left(\frac{b_{1}+b_{2}}{2}+1\right) !} .
\end{aligned}
$$

Используя данную форму записи, мы можем изучать асимптотические свойства кратностей при $p \rightarrow \infty$. Функция кратности для $\mathfrak{g}=A_{2}$ в координатах $\left\{b_{j}\right\}$ имеет следующий главный член:

$$
\left.M_{A_{2}}\left(p ; b_{1}, b_{2}\right)\right|_{p \rightarrow \infty} \sim \frac{b_{2}+1}{\left(\frac{b_{1}-b_{2}}{2}\right) !\left(\frac{b_{1}+b_{2}}{2}+1\right) !} p^{b_{1}} .
$$

Важно также знать, какой из неприводимых подмодулей $L^{\nu}$ имеет максимальную кратность в разложении $\left(L^{\omega_{1}}\right)^{\otimes p}=\sum_{\nu} M(\nu, p) L^{\nu}$. Возникает вопрос: как его координаты $\left(\nu_{\max }+\rho\right)_{i}$ зависят от $p$ ? Проблема в том, что даже в простейшем случае зависимость $\left(\nu_{\max }+\rho\right)_{i}(p) \equiv a_{\max , i}(p)$ нерегулярна. Например, в случае алгебры $A_{1}$ имеем $a_{\max }(20)=5, a_{\max }(25)=6$, а при $p=30$ максимум возвращается в предыдущую координату: $a_{\max }(30)=5$. Соответствующие кратности равны $M_{A_{1}}(20 ; 5)=48450, M_{A_{1}}(25 ; 6)=1225785$ и $M_{A_{1}}(30 ; 5)=33266625$. В следующем разделе мы обсудим особенности этой проблемы и объясним указанную выше нерегулярность.

\section{3. МАКСИМУМЫ ФУНКЦИИ КРАТНОСТИ. ПРОСТЕЙШИЙ СЛУЧАЙ}

Перепишем функцию кратности для алгебры $A_{1}$ через Г-функцию:

$$
M_{A_{1}}(p ; a)=\frac{\Gamma(p+1) a}{\Gamma\left(\frac{p-a+3}{2}\right) \Gamma\left(\frac{p+a+3}{2}\right)} .
$$




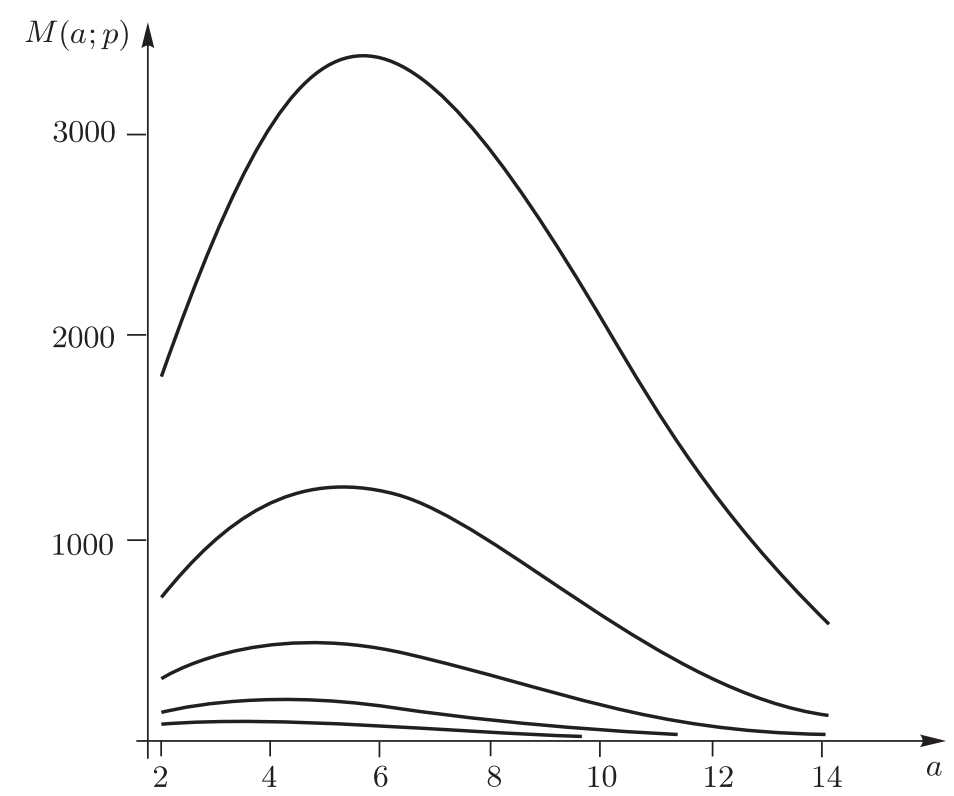

Рис. 1. Случай алгебры $A_{(1)}$. Максимум функции кратности движется в направлении старших весов $l$ (последовательно снизу вверх кривые отвечают $p=10,15,20,25,30$; каждая кривая масштабирована множителем 1/10 по отношению к предыдущей.)

Теперь можно определить, каким образом координаты точки $\max (M(p ;\{a\}))$ зависят от $p$. Например, в случае $\mathfrak{g}=A_{1}$ функция $a_{\max }(p)$ гладкая, и ее значение растет с ростом старшего веса $(p \omega)$ (см. рис. 1). Этот процесс описывает появление точек, соответствующих решетке весов $P_{A_{1}}$, в окрестности движущейся точки $a_{\max }(p)$ (см. рис. 2). Тонкие сплошные линии на рисунке показывают траекторию изменения окрестности с ростом $p$, а звездочки указывают точки решетки $P_{A_{1}}$, которые являются координатами старшего веса, $\left(\nu_{\max }+\rho\right)(p) \equiv a_{\max }(p)$ в разложении (1). Последовательность точек с весом $a_{\max }(p)$ нерегулярна, но основу процесса (при малых $p$ ) составляют регулярная и гладкая функция кратности $M_{A_{1}}(p ; a)$ и ее производные.

\section{4. МАКСИМУМЫ ФУНКЦИИ КРАТНОСТИ РАНГА 2}

Аналогичным образом введем гладкие функции кратности для $A_{2}$ и $B_{2}$ :

$$
\begin{aligned}
& M_{A_{2}}\left(p ;\left\{a_{1}, a_{2}\right\}\right)=\frac{\Gamma(p+1) a_{1} a_{2}\left(a_{1}+a_{2}\right)}{\Gamma\left(\frac{p-\left(a_{1}+2 a_{2}\right)+6}{3}\right) \Gamma\left(\frac{p+\left(2 a_{1}+a_{2}\right)+6}{3}\right) \Gamma\left(\frac{p+\left(-a_{1}+a_{2}\right)+6}{3}\right)}, \\
& M_{B_{2}}\left(p ;\left\{a_{1}, a_{2}\right\}\right)=\frac{\Gamma(p+1) \Gamma(p+3) a_{1} a_{2}\left(a_{1}+a_{2}\right)\left(a_{1}-a_{2}\right)}{4 \Gamma\left(\frac{p+a_{1}+5}{2}\right) \Gamma\left(\frac{p+a_{2}+5}{2}\right) \Gamma\left(\frac{p-a_{1}+5}{2}\right) \Gamma\left(\frac{p-a_{2}+5}{2}\right)} .
\end{aligned}
$$

Как мы уже видели в предыдущем примере, наша задача состоит в нахождении максимумов функций (2), (3) и зависимостей этих максимумов от $p$. 


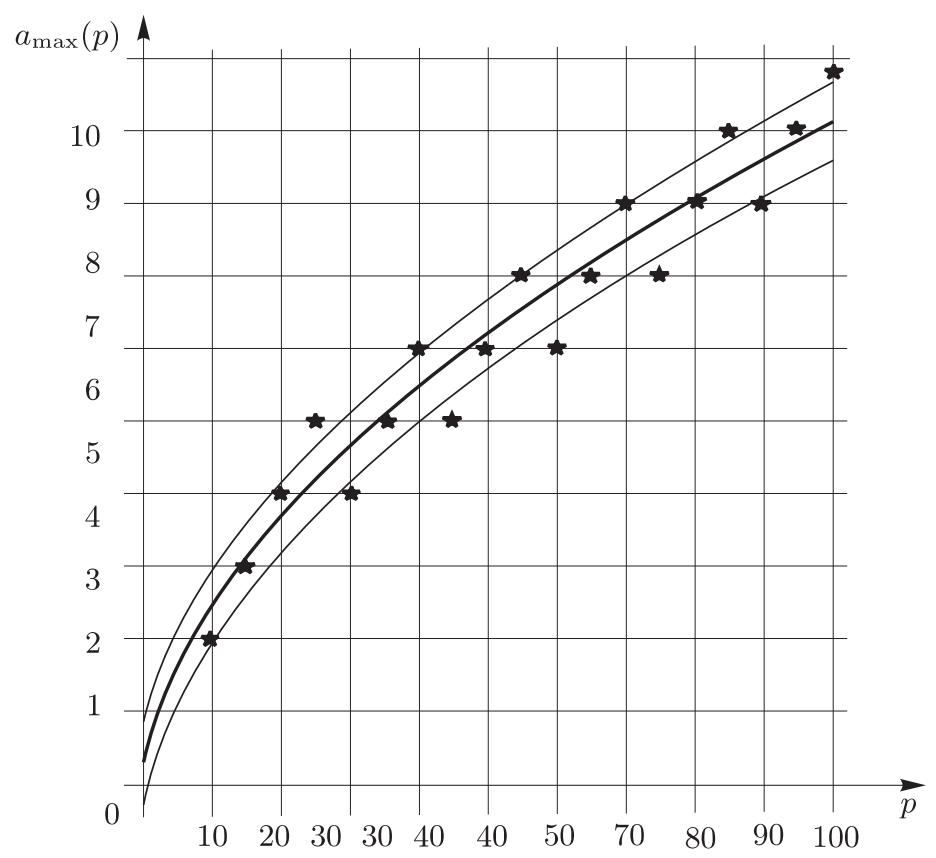

Рис. 2. Случай алгебры $A_{(1)}$. Максимумы функции кратности "активируют" ближайшие целочисленные точки решетки весов.

Рассмотрим сначала случай $\mathfrak{g}=A_{2}$. Координаты $l_{i}(p):=a_{i \max }(p)$ подчиняются следующим уравнениям:

$$
\begin{gathered}
\frac{2}{3} \boldsymbol{\Psi}\left(\frac{p-\left(l_{1}+2 l_{2}\right)+6}{3}\right)-\frac{1}{3} \boldsymbol{\Psi}\left(\frac{p+\left(2 l_{1}+l_{2}\right)+6}{3}\right)- \\
-\frac{1}{3} \boldsymbol{\Psi}\left(\frac{p+\left(-l_{1}+l_{2}\right)+6}{3}\right)+\frac{1}{l_{2}}+\frac{1}{l_{1}+l_{2}}=0 \\
\frac{2}{3} \boldsymbol{\Psi}\left(\frac{p+\left(2 l_{1}+l_{2}\right)+6}{3}\right)-\frac{1}{3} \boldsymbol{\Psi}\left(\frac{p-\left(l_{1}+2 l_{2}\right)+6}{3}\right)- \\
-\frac{1}{3} \boldsymbol{\Psi}\left(\frac{p+\left(-l_{1}+l_{2}\right)+6}{3}\right)-\frac{1}{l_{1}}-\frac{1}{l_{1}+l_{2}}=0
\end{gathered}
$$

где $\boldsymbol{\Psi}$ - логарифмическая производная функции Г. Для больших $p$ эти уравнения можно упростить, и они принимают вид

$$
\begin{aligned}
& \exp \left\{-3\left(\frac{1}{l_{2}}+\frac{1}{l_{1}+l_{2}}\right)\right\}=\frac{\left(p-\left(l_{1}+2 l_{2}\right)+6\right)^{2}}{\left(p+\left(2 l_{1}+l_{2}\right)+6\right)\left(p+\left(-l_{1}+l_{2}\right)+6\right)}, \\
& \exp \left\{-3\left(\frac{1}{l_{1}}+\frac{1}{l_{1}+l_{2}}\right)\right\}=\frac{\left(p-\left(l_{1}+2 l_{2}\right)+6\right)\left(p+\left(-l_{1}+l_{2}\right)+6\right)}{\left(p+\left(2 l_{1}+l_{2}\right)+6\right)^{2}} .
\end{aligned}
$$


Введем в качестве новой координаты $c$ расстояние между началом координат и точкой $\left(l_{1}, l_{2}\right)$. В этих обозначениях приближенное решение выглядит так:

$$
\begin{gathered}
l_{2}=l_{1}-\frac{1}{2} \\
c=\sqrt{\frac{3.44+p}{0.67}}-0.01 .
\end{gathered}
$$

Это означает, что максимум с ростом $p$ движется вдоль корня $\alpha_{3}$, центральной линии на $C_{A_{2}}^{(0)}$. Заметим, что это приближение хорошо работает при $p \gg 1$ (см. рис. 3 ).

Можно также задать вопрос, в какой точке весового пространства число состояний максимально. Чтобы ответить на этот вопрос, рассмотрим произведение функции кратности и размерности соответствующего модуля:

$$
M_{A_{2}}\left(p ;\left\{a_{1}, a_{2}\right\}\right) \operatorname{dim} L^{\left[a_{1}, a_{2}\right]}=M_{A_{2}}\left(p ;\left\{a_{1}, a_{2}\right\}\right) a_{1} a_{2}\left(a_{1}+a_{2}\right) .
$$

Здесь максимум с ростом $p$ также движется вдоль центральной линии $l_{2} \approx l_{1}-0.82$ на $C_{A_{2}}^{(0)}$ (см. рис. 4).

Соответствующие расчеты для $B_{2}$ приводят к следующим соотношениям для координат $l_{1}, l_{2}$ максимума функции кратности:

$$
\begin{aligned}
& \frac{1}{l_{1}}+\frac{1}{l_{1}+l_{2}}+\frac{1}{l_{1}-l_{2}}+\frac{1}{2} \boldsymbol{\Psi}\left(\frac{p-l_{1}+5}{2}\right)-\frac{1}{2} \boldsymbol{\Psi}\left(\frac{p+l_{1}+5}{2}\right)=0, \\
& \frac{1}{l_{2}}+\frac{1}{l_{1}+l_{2}}+\frac{1}{l_{1}-l_{2}}+\frac{1}{2} \boldsymbol{\Psi}\left(\frac{p-l_{2}+5}{2}\right)-\frac{1}{2} \boldsymbol{\Psi}\left(\frac{p+l_{2}+5}{2}\right)=0 .
\end{aligned}
$$

На этот раз аргументы $l_{i}$ функции $M_{B_{2}}^{\max }\left(p ;\left\{l_{1}, l_{2}\right\}\right)$ (см. выражение $\left.(3)\right)$ являются ортонормированными координатами в $P_{B_{2}}$. Для больших $p$ снова удобно ввести параметр $c$ (расстояние между началом координат и точкой $\left.\left(l_{1}, l_{2}\right)\right)$. Приближенные решения системы (9) выглядят так (см. рис. 5):

$$
l_{2}=0.049+0.412 l_{1}, \quad c=-0.5+2 \sqrt{p+3.13} .
$$

Отметим, что в этом случае координаты $l_{1}, l_{2}$ снова удовлетворяют одному линейному условию, а второе уравнение является параболическим.

Для числа состояний, т. е. для функции кратности, умноженной на размерность модуля со старшим весом $\nu=\left(l_{1}-1\right) \omega_{1}+\left(l_{2}-1\right) \omega_{2}$, результат аналогичен (см. рис. 6$)$ :

$$
l_{2}^{\mathrm{st}}=0.15+0.53 l_{1}^{\mathrm{st}}, \quad c^{\mathrm{st}}=-1.46+3.57 \sqrt{7.14 p+19.90} .
$$

Покажем, как работают полученные соотношения. Вернемся к случаю $\mathfrak{g}=A_{2}$ и найдем решение с максимальной кратностью для $p=100$. Соотношение (7) приводит к параметру (расстоянию) $c=12.41$. Это означает, что $l_{1}+l_{2}=2 c / \sqrt{3}=14.34$. Принимая во внимание уравнение (6), получим координаты максимальной "кратности" для $p=100: l_{1}=7.42, l_{2}=6.92$. Теперь мы можем воспользоваться гладкостью функции $M_{A_{2}}\left(p ;\left\{a_{1}, a_{2}\right\}\right)$ и прийти к выводу, что максимальный коэффициент кратности $m_{A_{2}}\left(p ;\left\{a_{1}, a_{2}\right\}\right)$ соответствует точке $\left(a_{1}, a_{2}\right)$ с целочисленными координатами, ближайшей к точке $\left(l_{1}, l_{2}\right)$. Мы должны вспомнить классы конгруэнтности, для алгебры $A_{2}$ их три. Для первого класса получим точку $(8,7)$, для второго - точку $(8,6)$ и для третьего - точку $(7,7)$. Эта ситуация показана на рис. 7,8 и 9 соответственно. Соответствующие индексы Дынкина для неприводимых модулей $L^{\left[m_{1}, m_{2}\right]}$ в $\left(L^{\omega_{1}}\right)^{\otimes 100}$ суть $[7,6],[7,5]$ и $[6,6]$. 


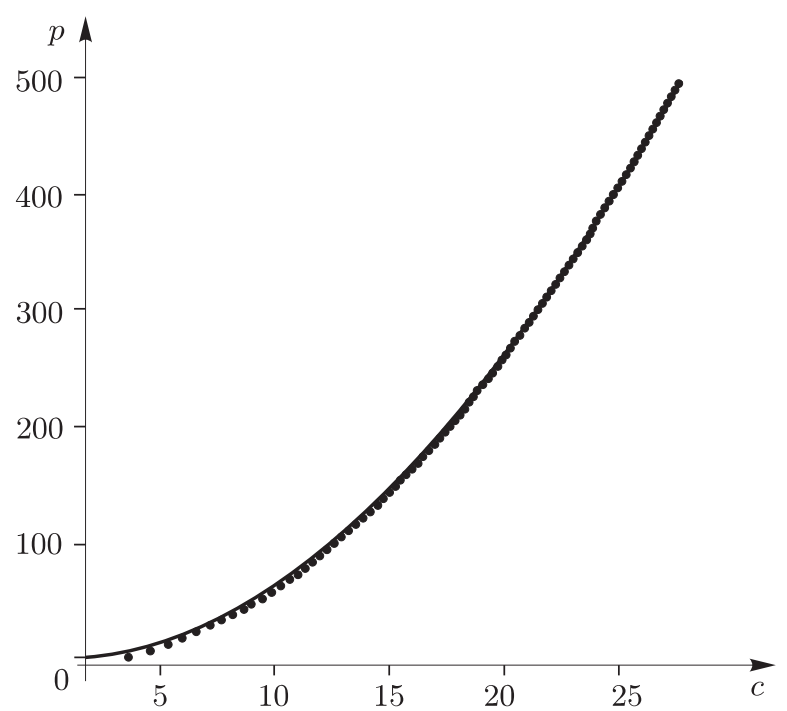

Рис. 3. Случай алгебры $A_{(2)}$. Максимум функции кратности в зависимости от расстояния $c$ от центра симметрии Вейля.

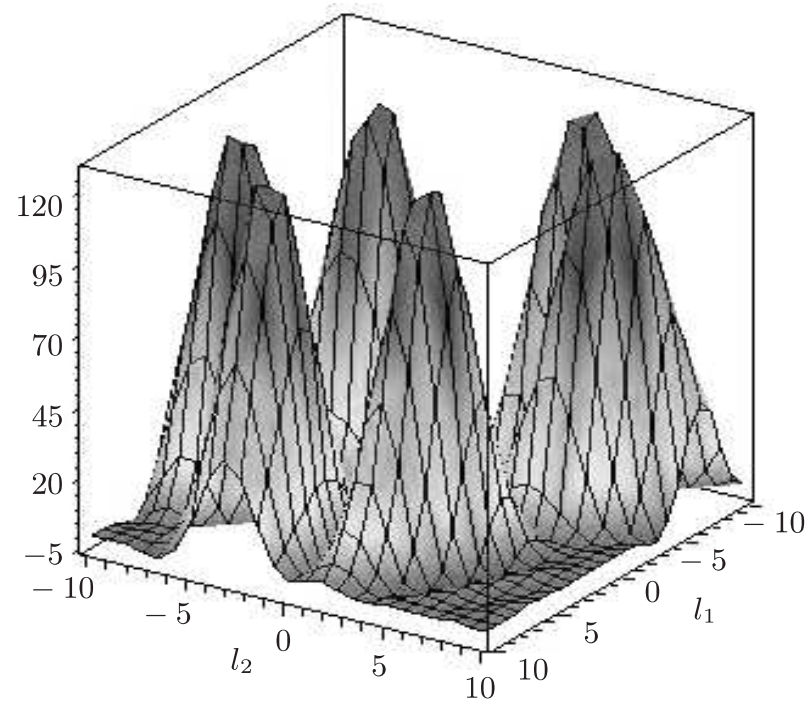

Рис. 4. Случай алгебры $A_{(2)}$. Произведение функции кратности $M(\nu)$ и размерности соответствующего модуля $L^{\nu}$ (масштабировано множителем $10^{2}$ ). Фундаментальная камера Вейля содержит фронтальный пик. 


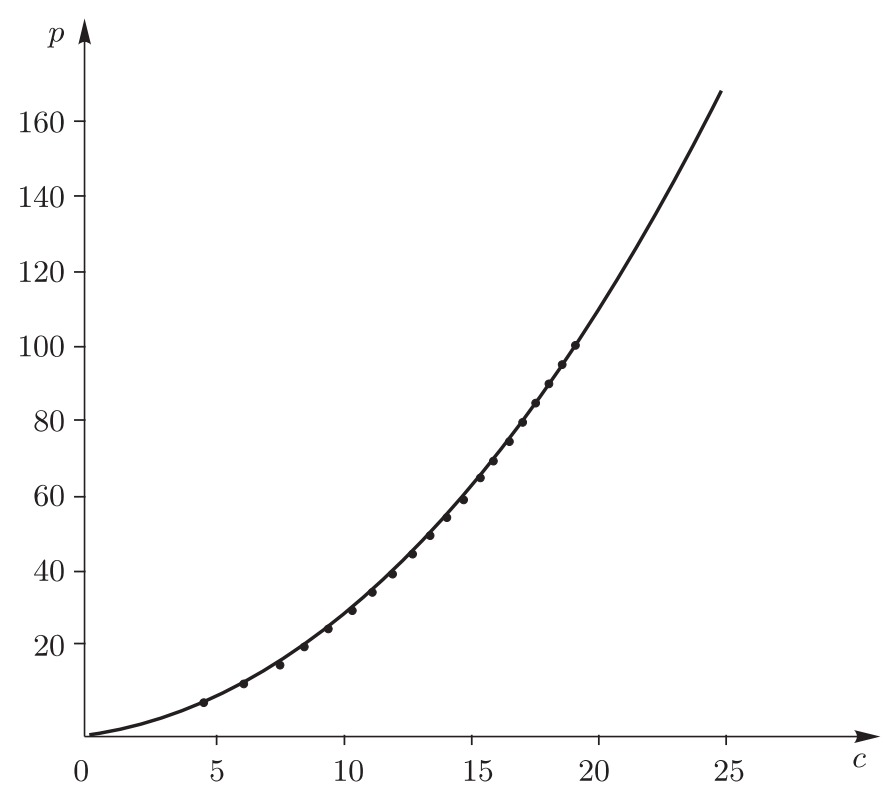

Рис. 5. Случай алгебры $B_{(2)}$. Движение максимума функции кратности хорошо описывается кривой $c=-0.5+2 \sqrt{p+3.13}$.

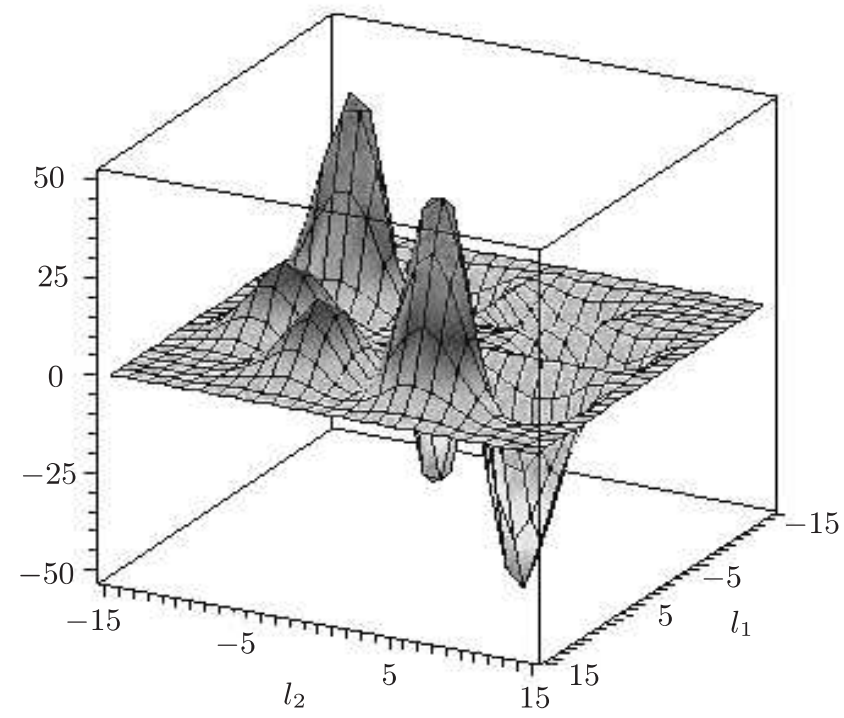

Рис. 6. Случай алгебры $B_{(2)}$. Функция кратности $M(\nu)$, умноженная на размерность соответствующего модуля $L^{\nu}$ (масштабировано множителем $10^{4}$ ). Фундаментальная камера Вейля содержит фронтальный пик, некоторые пики отрицательны, в отличие от случая $A_{(2)}$. 


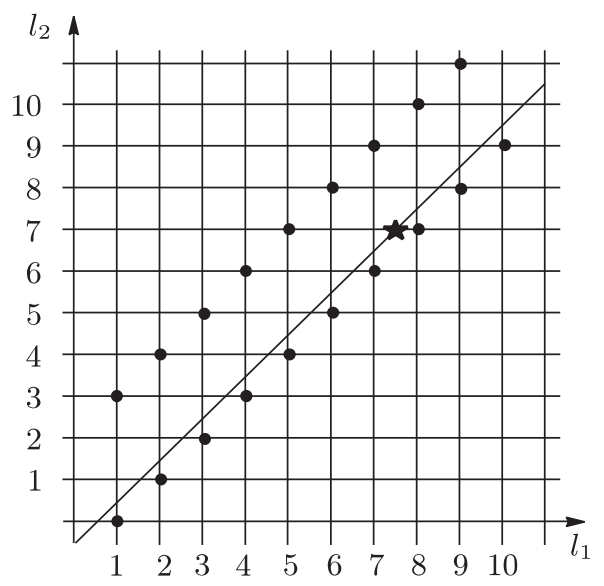

Рис. 7. Алгебра $A_{(2)}$. Максимумы функции кратности и точки первого класса конгруэнтности на решетке. Здесь и на последующих рисунках максимум для $p=100$ обозначен звездочкой.

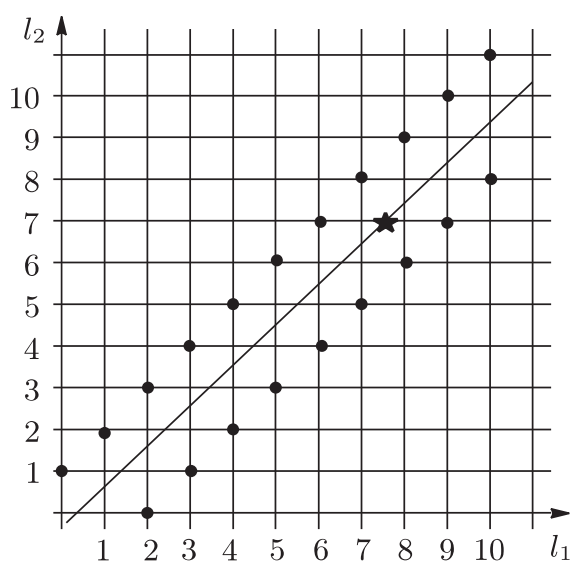

Рис. 8. Алгебра $A_{(2)}$. Точки второго класса конгруэнтности.

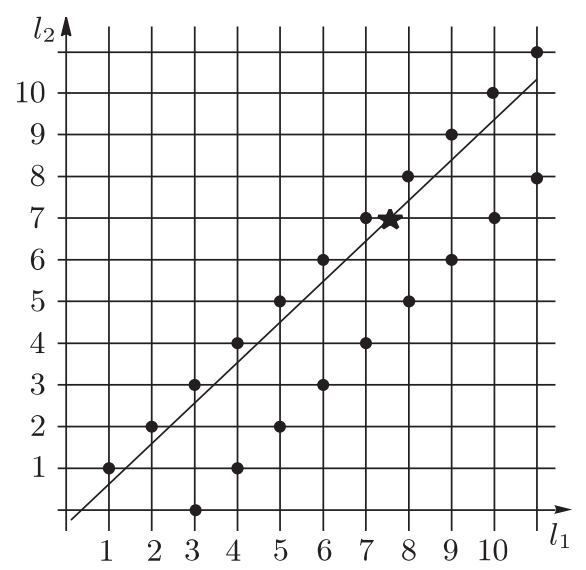

Рис. 9. Алгебра $A_{(2)}$. Точки третьего класса конгруэнтности.

\section{5. ВЫВОДЫ}

Мы продемонстрировали, что функция кратности $M_{\mathfrak{g}}\left(p ;\left\{a_{i}\right\}\right)$ является удобным инструментом для изучения свойств разложений тензорных степеней. Ее можно использовать как для нахождения предельных свойств различных кратностей старших весов, так и для исследования "динамики" (по тензорной степени $p$ ) веса с максимальной кратностью.

Возможность построения функций кратности для других модулей (не только для фундаментальных модулей минимальной размерности) является проблемой огромной важности. 
Благодарности. Автор выражает благодарность организаторам Международного семинара "Классические и квантовые интегрируемые системы" (CQIS-2012) за гостеприимство. Работа частично поддержана РФФИ (грант № 12-01-00207).

\section{Список литературы}

[1] S. Kumar, "Tensor product decomposition", Proceedings of the International Congress of Mathematicians (Hyderabad, August 19-27, 2010), v. 3, eds. R. Bhatia, A. Pal, G. Rangarajan, V. Srinivas, M. Vanninathan, Hindustan Book Agency, New Delhi, 2010, 1226-1261.

[2] А.Н. Кириллов, Н. Ю. Решетихин, Зап. научн. сем. ПОМИ, 205 (1993), 30-37.

[3] M. Kleber, Internat. Math. Res. Notices, 4 (1997), 187-201, arXiv: q-alg/9611032.

[4] G. Hatayama, A. Kuniba, M. Okado, T. Takagi, Y. Yamada, "Remarks on fermionic formula", Recent Developments in Quantum Affine Algebras and Related Topics, Proceedings of the International Conference on Representations of Affine and Quantum Affine Algebras and Their Applications (Raleigh, NC, USA, May 21-24, 1998), Contemporary Mathematics, 248, eds. N. Jing, K. Misra, AMS, Providence, RI, 1999, 243-291, arXiv: math/9812022.

[5] G. Hatayama, A. Kuniba, M. Okado, T. Takagi, "Combinatorial $R$-matrices for a family of crystals: $B_{n}(1), D_{n}(1), A_{2 n}(2)$ and $D_{n+1}(2)$ cases", Physical Combinatorics, Proceedings of a Workshop (Kyoto, Japan, January 29 - February 2, 1999), Progress in Mathematics, 191, eds. M. Kashiwara, T. Miwa, Birkhäuser, Boston, 2000, 105-139, arXiv: math/0012247.

[6] G. Hatayama, A. Kuniba, M. Okado, T. Takagi, Z. Tsuboi, "Paths, crystals and fermionic Formulae", MathPhys Odyssey 2001, Integrable models and beyond. In honor of Barry M. McCoy, dedicated to his 60th birthday, Progress in Mathematical Physics, 23, eds. M. Kashiwara, T. Miwa, Birkhäuser, Boston, 2002, 205-272, arXiv: math/0102113.

[7] M. Kleber, Finite dimensional representations of quantum affine algebras, Ph. D. Thesis, University of California, Berkeley, 1998, arXiv: math/9809087.

[8] P. P. Kulish, V.D. Lyakhovsky, O. V. Postnova, J. Phys. A, 343:1 (2012), 012070, 9 pp.

[9] P. P. Kulish, V.D. Lyakhovsky, O. V. Postnova, J. Phys. A, 343:1 (2012), 012095, 7 pp.

[10] P. P. Kulish, V.D. Lyakhovsky, O. V. Postnova, J. Phys. A, 346:1 (2012), 012012, 15 pp., arXiv: 1106.2002.

[11] П. П. Кулиш, В. Д. Ляховский, О.В.Постнова, ТМФ, 171:2 (2012), 283-293. 